\title{
FLUCTUACIÓN POBLACIONAL DE LA MOSCA MINADORA Liriomyza huidobrensis Blanchard Y SUS PARA- SITOIDES EN EL CULTIVO DE HABA EN LA ESTACIÓN EXPERIMENTAL DE LA UNIVERSIDAD NACIONAL DEL CENTRO DEL PERÚ-HUANCAYO*
}

\author{
Lindo Gutarra, Efraín Bernabé y Paucarchuco Tovar, Teodoro² \\ Facultad de Agronomía de la Universidad Nacional del Centro del Perú
}

\begin{abstract}
RESUMEN
El estudio de la fluctuación poblacional de la mosca minadora Liriomyza huidobrensis Blanchard y sus parasitoides fue realizado en el Valle del Mantaro en la Estación Experimental del Mantaro de la Universidad Nacional del Centro del Perú-Jauja; ubicado a 11049>00» latitud sur y 75022>05» longitud oeste; al sur de la ciudad de Huancayo a una distancia de $32 \mathrm{~km}$. Para la toma de muestra, el campo se dividió en 5 zonas excluyendo en el margen una franja de 5 metros para evitar el efecto de borde. En la fase de campo se determinó el número de adultos posados en la mata de $20 \mathrm{~cm}$ de diámetro, se colectaron al azar 5 hojas por zona, completando 25 hojas/evaluación, las que se colocaban en papel kraft 20, y se trasladaron al laboratorio. Los datos de temperatura promedio (X1), humedad relativa (X2), horas de sol (X3) y precipitación (X4) fueron obtenidos del Observatorio Metereológico de Jauja, ubicado a $11^{\circ} 47^{\prime} 01^{\prime \prime}$ latitud sur, 75²8'28' longitud oeste, $3322 \mathrm{msnm}$ y a una distancia de $12 \mathrm{~km}$ al sur del Mantaro. En la fase de laboratorio, en base a las 25 hojas por evaluación, se registró el número de adultos de ectoparasitoides, total de foliolos, minados, puparios recuperados, puparios viables, puparios no viables, adultos de $L$. huidobrensis, endoparasitoides emergidos y diapausales. La más alta incidencia de adultos de Liriomyza huidobrensis ocurrió en los meses de octubre y noviembre en la fase fenológica de floración y fructificación. El registro más alto fue de 495 adultos en $20 \mathrm{~cm}$ de diámetro en la evaluación del 22 de octubre. La mayor riqueza de especies se recupera a partir del 24 de octubre hasta el final de la campaña con siete especies, compuesta de 4 endoparasitoides (Halticoptera arduine, Chrysocharis sp. Chrysocharis phytomyzae y Phaedrotoma sp. ) y 3 ectoparasitoides (Diglyphus begini, D. websteri y Closterocerus cinctipennis). El más alto porcentaje de endoparasitismo fue alcanzado el 26 de noviembre, mientras el mayor número de individuos endoparasitoides fue registrado el 05 de noviembre con 358 individuos que representó un 52.6\%. El mayor porcentaje de endoparasitismo se registró entre el mes de noviembre en la fase de fructificación y maduración del cultivo. El parásitoide más abundante fue Halticoptera arduine alcanzando sus mayores porcentaje de infestación en el macollamiento registrando un 50\% en el mes de setiembre y el mayor número de individuos (258) fue registrado el 05 de noviembre que representan el 37,9\% de parasitismo en la fase de fructificación. Halticoptera arduine presenta un estado diapausal, de la recuperaciones del 10 y 15 de diciembre con una duración de 4 a 4,5 meses. El ectoparasitoide más abundante fue Diglyphus begini, con una población máxima de 56 individuos recuperados de la evaluación del 15 de octubre en la fase de floración.
\end{abstract}

Palabra clave: mosca minadora.

\footnotetext{
* Este trabajo de investigación fue recibido el 20/03/2005 retornado para su revisión 26/10/2009 y aprobado para su publicación 16/11/2009

1Email: lindoefrain@hotmail.com

2Email: tpaucarchuco@uncp.edu.pe
} 


\title{
POPULATION FLUCTUATIONS OF THE LEAFMINER FLY Liriomyza huidobrensis Blanchard AND THEIR PARASITOIDS IN GROWING BEANS IN THE EXPERIMENTAL STATION OF THE UNIVERSIDAD NACIONAL DEL CENTRO DEL PERU-HUANCAYO
}

\begin{abstract}
The study of population fluctuation of the pea leaf miner Liriomyza huidobrensis Blanchard and their parasitoides in "red UNCP" bean fields in the Mantaro Valley, was carried out in the experimental fields of the Mantaro Experimental Station of the Universidad Nacional del Centro del Peru; located at $11^{\circ} 49^{\prime} 00$ South latitude and $75^{\circ} 22^{\prime} 05$ West longitude. For sampling, the field was divided in 5 areas excluding at the margins a fringe of 5 meters to avoid the border effects. In the field phase the number of adults posed on the plant at $20 \mathrm{~cm}$. diameter, 5 leaves were collected at random by area, completing 25 leaves/evaluation, those were placed in a paper kraft 20 bag and were taken to the laboratory. In the laboratory phase, based on 100 leaves evaluated we registered the number of adult ectoparasitoids, total of leaflets, mined leaflets, recovered puparia, viable puparia, non viable puparia, adults of $L$. huidobrensis, emerged endoparasitoids and diapausing individuals. The data of mean temperature (X1), relative humidity (X2), hours of sun light (X3) and precipitation (X4) were obtained at the Observatorio Metereológico of Jauja, located at $11^{\circ} 47^{\prime} 01^{\prime}$ "South latitude, $75^{\circ} 28^{\prime} 28$ " West longitude, $3322 \mathrm{msnm}$. The highest incidence of adults of Liriomyza huidobrensis Blanchard occurred in the months of october, November and december in the fruition phase and maturation. The highest density of adults 495 was recorded in the evaluation of october 22. The highest species richness recovers starting from october 24 until the end of the campaign with seven species, made up of 4 endoparasitoids (Halticoptera arduine, Chrysocharis sp. Chrysocharis phytomyzae and Phaedrotoma sp. ) and 3 ectoparasitoids (Diglyphus begini, D. websteri and Closterocerus cinctipennis). The highest endoparasitism percentage was reached on november 26 , while the highest number of individual endoparasitoids was registered on november 5 with 358 individuals that represented $52,6 \%$. The highest endoparasitism percentage was registered between october and november in the fruition phase and maturation of the crop. The most abundant parasitoid was Halticoptera arduine reaching its highest percentage at beginning the macoll registering $50 \%$ in september and the largest number of individuals (258) on november 5 which represented $37,9 \%$ of parasitism in the fruition phase. Halticoptera arduine presents a slight diapause stage, of the recoveries from december to may in the phase and maturation of the crop, registered one diapausal period between 4 and 4,5 months. The most abundant ectoparasitoid was Diglyphus begini, with a maximum population density of 56 recovered individuals on the evaluation of october 15 in the flower phase.
\end{abstract}

Key words: mine life 


\section{INTRODUCCIÓN}

El Vicia faba es uno de los cultivos alimenticios de gran importancia en el área andina del Perú. Es valorada por sus cualidades alimenticias y nutricionales de la semilla que contiene $27,7 \%$ de proteína, 1,5\% de grasa, $3,2 \%$ de ceniza y $11,5 \%$ de humedad. La producción es afectada por una serie de factores abióticos y bióticos. Entre los bióticos están las plagas que con frecuencia afectan al cultivo en el Valle del Mantaro una de las plagas es la "mosca minadora" Liriomyza huidobrensis Blanchard. En el Valle del Mantaro aquí, el control de L. huidobrensis se basa en la utilización de diferentes insecticidas órgano-sintéticos, los cuales son usados por la gran mayoría de agricultores en forma empírica. Como consecuencia este insecto ha adquirido cierta tolerancia a los diferentes productos tradicionales. Por lo cúal es necesario determinar la fluctuación de la mosca minadora y determinar la fluctuación poblacional de la riqueza de los parasitoides.

\section{MATERIAL Y MÉTODOS}

Los muestreos se iniciaron el 20 de agosto hasta el 17 de diciembre del 2 004. Para el muestreo el campo se dividió en 5 sectores, donde se evaluó 25 matas al azar, teniendo en cuenta que en cada evaluación se abarque todo el sector. En cada una de las evaluaciones a nivel de campo se registró: Número de moscas adultas por planta, porcentaje de infestación y en laboratorio se determinó: número de puparios de mosca minadora más pupas de parasitoides, número de moscas emergidas, número de parasitoides emergidos, número de parasitoides en estado de diapausa, número de puparios no eclosionados y porcentaje de parasitismo.

Para un análisis estadístico, se utilizó la regresión y correlación múltiple considerando las siguientes variables: variable dependiente: $(Y)$ : número de moscas adultas por 25 plantas, porcentaje de infestación, porcentaje de parasitismo, porcentaje de parasitismo de cada especie importante .y las variables independientes: se consideró, temperatura promedio (X1), humedad relativa promedio (X2), horas de sol promedio (X3), precipitación (X4).

\section{RESULTADOS Y DISCUSIÓN}

\section{Fluctuación poblacional de adultos de $L$. huidobrensis}

En el gráfico 1, se presentan los registros promedio de adultos durante las 18 evaluaciones en haba var. roja UNCP.

Del gráfico se aprecia que desde el inicio del desarrollo del cultivo (20 de agosto) hasta la séptima evaluación (1 de octubre) se registró la población más baja de adultos de todo el período de observaciones con un total que fluctuó entre 15 a 109 individuos / 25 matas. Sin embargo a partir de la octava evaluación, en la floración (15 octubre), se registró un incremento de la población (259 individuos), llegando el pico más alto el 19 de noviembre, luego decreció en la catorceava evaluación, debido al desarrollo fenológico de la planta.

A partir del 14 de noviembre, en la fructificación, el número de adultos de la mosca minadora ha decrecido observándose una mínima población en las dos últimas evaluaciones, es decir, en la maduración de la planta. Estas observaciones coinciden con los resultados obtenidos por Chuquillanqui (1998) quien registró un menor número de adultos por planta (7,5 adultos) en la madurez fisiológica del cultivo de haba, asímismo Mujica (1 999) en frijol registró un menor número de larvas en la fase de maduración del cultivo; sin embargo Ccasa y Mamani (1995) registraron al final de campaña en Arequipa en papa un incremento en el número de adultos por planta (5,3 adultos).

Las mayores poblaciones se registraron en los meses de octubre y noviembre (gráfico 1) en los cuales la temperatura promedio osciló entre $12.1 \mathrm{a} 12.3^{\circ} \mathrm{C}$.

\section{Total y porcentaje de foliolos minados/25 hojas.}

En el gráfico 2 se presentan los resultados del total y porcentaje de foliolos minados en 25 hojas evaluadas. Se aprecia a partir del 20 de agosto un porcentaje de infestación mínima esta tendencia se mantiene hasta el 1 de octubre alcanzando solo 1,84\% (en 326 foliolos evaluados), cuando la temperatura promedio del medio ambiente se incrementó desde $11.2^{\circ} \mathrm{C}$ a $12.4^{\circ} \mathrm{C}$. 
Al inicio de la fructificación (29 de octubre) el porcentaje de infestación se incrementó drásticamente a 41.50\% de 400 foliolos evaluados y este daño fue mayor conforme transcurrió el desarrollo del cultivo hasta llegar a un máximo de $99.47 \%$ (567 foliolos) evaluados en la fase de maduración.

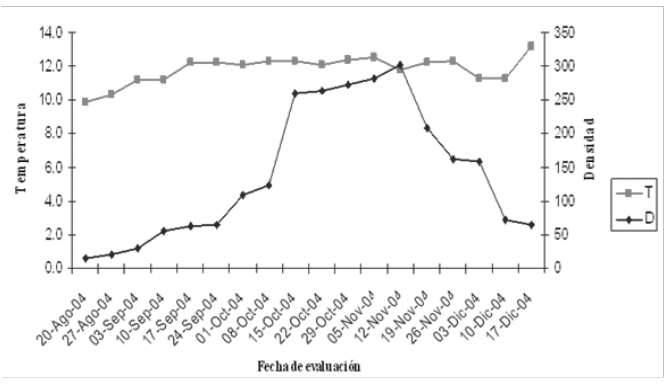

Gráfico 1. Fluctuación poblacional de la mosca minadora

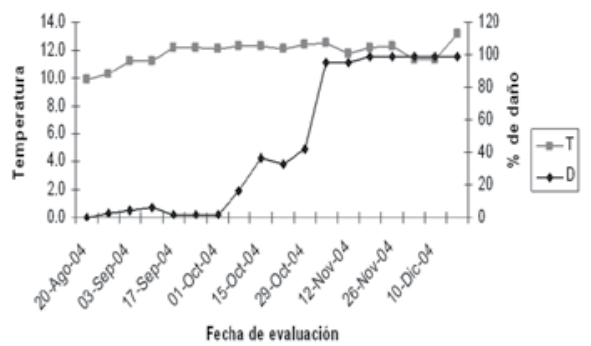

Gráfico 2.Porcentaje de infestación de larvas de la mosca minadora.

De los gráficos 1 y 2 se observan que conforme se incrementó la población de adultos de la mosca minadora, el número de foliolos y porcentaje de daño a partir del 29 de octubre, se incrementaron y a partir del 10 de diciembre cuando declinó la población de adultos, el porcentaje de infestación se mantuvo, debido a que las plantas ya no tienen el mismo crecimiento y no emiten nuevas hojas; estas observaciones coinciden con los citados por Molina (1990) quien registró los mayores niveles de infestación en la etapa de floración y tuberización en el cultivo de papa.

\section{Total y porcentaje de puparios no viables}

Los resultados sobre el total de puparios no viables de $L$. huidobrensis recuperados durante las 18 evaluaciones en la estación del Mantaro se presentan en el cuadro 1.

Desde la segunda hasta la quinta evaluación (setiembre, 17) no se registró puparios no viables, a partir de la sexta evaluación hasta la última evaluación se registraron puparios no viables en distintos porcentajes y de acuerdo a la evaluación estas cifras fueron heterogéneas a pesar de no existir aplicaciones de insecticidas. El más alto número de puparios no viables fue registrado el 05 de noviembre con 15,9\% que representan 108 puparios y el más bajo se registró el 10 de octubre con un porcentaje de 7,1\% (2 puparios). 
Los porcentajes de puparios no viables obtenidos en este campo son inferiores a los registrados en la costa sobre el cultivo de papa, así Molina (1990) indica un rango de 38 a 81\%, en tanto que en el presente trabajo se registró un rango de 0 a 18.6\%, sin embargo en cultivos de alta presión de insecticidas la viabilidad disminuye, así Lizarraga y Mujica (1991) registraron una viabilidad del 49\%.

Cuadro 1. Número de puparios viables y no viables, adultos de $L$. huidobrensis Blanchard y endoparasitoides recuperados de foliolos de haba var. Roja UNCP. En la estación experimental del Mantaro. Jauja. Periodo: agosto-diciembre, 2 004. Huancayo-Perú.

\begin{tabular}{|c|c|c|c|c|c|c|c|c|c|}
\hline \multirow[t]{2}{*}{$\begin{array}{c}\text { Fechas de } \\
\text { evaluaciones }\end{array}$} & \multirow{2}{*}{$\begin{array}{l}\text { Puparios de L. } \\
\text { huidobrensis } \\
\text { recuperados }\end{array}$} & \multicolumn{2}{|c|}{$\begin{array}{l}\text { Puparios no } \\
\text { viables }\end{array}$} & \multicolumn{2}{|c|}{$\begin{array}{c}\text { Puparios de } L \text {. } \\
\text { huidobrensis } \\
\text { viables }\end{array}$} & \multicolumn{2}{|c|}{$\begin{array}{c}\text { Adultos de } L \text {. } \\
\text { huidobrenis } \\
\text { emergidos }\end{array}$} & \multicolumn{2}{|c|}{$\begin{array}{c}\text { Endo- } \\
\text { parasitoides }\end{array}$} \\
\hline & & $\mathrm{N}^{\circ}$ & $\%$ & $\mathrm{~N}^{\circ}$ & $\%$ & $\mathrm{~N}^{\circ}$ & $\%$ & $\mathrm{~N}^{\circ}$ & $\%$ \\
\hline 20-Ago-04 & 0 & 0 & 0 & 0 & 0 & 0 & 0 & 0 & 0 \\
\hline 27-Ago-04 & 5 & 0 & 0 & 5 & 100 & 3 & 60 & 2 & 40.0 \\
\hline 03-Sep-04 & 3 & 0 & 0 & 3 & 100 & 2 & 67 & 1 & 33.3 \\
\hline 10-Sep-04 & 7 & 0 & 0 & 7 & 100 & 6 & 85.7 & 1 & 14.3 \\
\hline 17-Sep-04 & 7 & 0 & 0 & 7 & 100 & 5 & 71 & 2 & 28.6 \\
\hline 24-Sep-04 & 30 & 3 & 10.0 & 27 & 90.0 & 7 & 23 & 20 & 66.7 \\
\hline 01-Oct-04 & 28 & 2 & 7.1 & 26 & 92.9 & 13 & 46.4 & 13 & 46.4 \\
\hline 08-Oct-04 & 112 & 10 & 8.9 & 102 & 91.1 & 47 & 42.0 & 55 & 49.1 \\
\hline $15-O c t-04$ & 117 & 11 & 9.4 & 106 & 90.6 & 67 & 57.3 & 39 & 33.3 \\
\hline 22-Oct-04 & 800 & 107 & 13.4 & 693 & 86.6 & 495 & 61.9 & 198 & 24.8 \\
\hline 29-Oct-04 & 600 & 87 & 14.5 & 513 & 85.5 & 230 & 38.3 & 283 & 47.2 \\
\hline 05-Nov-04 & 681 & 108 & 15.9 & 573 & 84.1 & 215 & 31.6 & 358 & 52.6 \\
\hline 12-Nov-04 & 400 & 86 & 21.5 & 314 & 78.5 & 168 & 42.0 & 146 & 36.5 \\
\hline 19-Nov-04 & 345 & 34 & 9.9 & 311 & 90.1 & 97 & 28.1 & 214 & 62.0 \\
\hline 26-Nov-04 & 342 & 22 & 6.4 & 320 & 93.6 & 41 & 12.0 & 279 & 81.6 \\
\hline 03-Dic-04 & 123 & 23 & 18.7 & 100 & 81.3 & 35 & 28.5 & 65 & 52.8 \\
\hline 10-Dic-04 & 134 & 12 & 9.0 & 122 & 91.0 & 36 & 26.9 & 86 & 64.2 \\
\hline 17-Dic-04 & 123 & 11 & 8.9 & 112 & 91.1 & 37 & 30.1 & 75 & 61.0 \\
\hline
\end{tabular}

Total y porcentaje de puparios viables de L. huidobrensis Blanchard.

Los resultados sobre el total de puparios viables de L. huidobrensis recuperados durante las 18 evaluaciones en la estación del Mantaro se presentan en el cuadro 1. 
En las primeras evaluaciones la viabilidad de las puparios fue de un $100 \%$, así en la segunda evaluación, setiembre, 17 se observa de las 5 puparios recuperadas todas emergieron y esta tendencia de viabilidad se registró hasta la quinta evaluación (17 de setiembre). A partir del 24 de setiembre la viabilidad fluctuó entre 78,5 a 92,9\%, observándose el porcentaje más alto de viabilidad dentro de este rango el 01 de octubre. Por otro lado la viabilidad durante las evaluaciones tuvo ligeras variaciones entre evaluaciones y evaluación sin embargo esta tendencia se mantuvo durante el desarrollo del cultivo. El porcentaje de puparios viables no varió en forma considerable en el desarrollo del cultivo, debido a que los puparios recuperados de foliolos tuvieron similar manejo en el laboratorio durante todo el periodo de observaciones.

\section{Total y porcentaje de adultos emergidos de $L$. huido- brensis Blanchard.}

Los resultados sobre el total de adultos emergidos de L. huidobrensis durante las 18 evaluaciones en la estación del Mantaro se presentan en el cuadro 1.

En la primera evaluación no se recuperaron puparios de las hojas, en la segunda evaluación (18 de julio) emergieron 3 adultos que representaron el $60 \%$ de los puparios recuperados (el mayor porcentaje registrado). En la onceava evaluación se registraron 495 adultos que representaron un $61,9 \%$ de los puparios recuperados y a partir de la doceava evaluación el porcentaje de emergencia de adultos de la mosca minadora disminuyó drásticamente debido a la presencia de los endoparasitoides. El menor número de adultos viables fue registrado el 01 de octubre con 13 adultos que representaron un $46,4.7 \%$. El porcentaje de adultos de mosca emergidos se basa en el total de puparios registradas en el laboratorio, en consecuencia de acuerdo a la fecha de evaluación esta cifra varía, y esta variación depende de la población de puparios.

\section{Parasitoides}

En el cuadro 2 se presenta una lista de las especies parasitoides de $L$. huidobrensis en el cultivo de haba var. roja UNCP en la siembra de agosto, 2004 en la estación experimental del Mantaro. Las ocho especies registradas corresponden a dos grupos diferentes: a) los ectoparasitoides idiobiontes, cuyas hembras para- lizan a la larva de la mosca minadora en las galerías de las hojas y luego ovipositan sobre o cerca de la larva hospedera. En este caso, las larvas de los parasitoides se alimentan externamente del hospedero y empupan en la galería. b) los endoparasitoides koinobiontes, en los cuales la hembra oviposita dentro de la larva hospedera y los adultos emergen del pupario de la mosca minadora.

Cuadro 2. Registro de los parasitoides de la mosca minadora campaña 2004-2005

\begin{tabular}{ll}
\hline \multicolumn{1}{c}{ GRUPO/FAMILIA } & \multicolumn{1}{c}{ ESPECIE } \\
\hline $\begin{array}{l}\text { ECTOPARASITOIDES } \\
\text { IDIOBIONTES } \\
\text { Eulophidae }\end{array}$ & $\begin{array}{l}\text { Diglyphus websteri (Crawford) } \\
\text { Diglyphus begini (Ashmead) } \\
\text { Closterocerus cinctipennis Ashmead }\end{array}$ \\
$\begin{array}{l}\text { ENDOPARASITOIDES } \\
\text { KOINOBIONTES }\end{array}$ & $\begin{array}{l}\text { Halticoptera arduine (Walker) } \\
\text { Eulophidae }\end{array}$ \\
Chysocharis sp. \\
Chysocharis phytomyzae (Brethes)
\end{tabular}

\section{Total de endoparasitoides emergidos y porcentaje de parasitismo.}

Los resultados sobre el total de adultos endoparasitoides emergidos de pupas de $L$. huidobrensis recuperados durante las 18 evaluaciones en la estación del Mantaro se presentan en el cuadro 3.

En la primera evaluación no se recuperó endoparasitoides. La segunda evaluación (27 de agosto, fase de crecimiento vegetativo) emergieron solo 2 endoparasitoides que representaron el $40 \%$ del parasitismo, en tanto que en la tercera y cuarta evaluación se recuperaron sólo un endoparasitoides. A partir de la sexta evaluación se incrementó el número de endoparasitoides, el 24 de setiembre en la floración se recuperaron 20 endoparasitoides que representaron el $66,7 \%$ de parasitismo. Esta tendencia fue heterogénea mantuvo durante el resto de la campaña, registrándose el mayor número de endoparasitoides el 05 de noviembre en la fructificación del cultivo, que representaron un $52.6 \%$ de parasitismo. El menor porcentaje de parasitismo observado fue en la 
floración (24 de octubre) se obtuvo 198 individuos que representaron un 24,8\% de parasitismo. Estas observaciones son similares a de Sánchez y Redolfi (1988), Molina (1990), Galantini y Redolfi (1992), Tejada (1998) para la Costa central y con Valverde y Paucarchuco (2000) en la sierra central.

Cuadro 3 Endoparasitoides recuperados de L. huidobrensis Blanchard de foliolos de haba var. Roja UNCP. en la estación experimental del Mantaro. Jauja. Periodo: agosto-diciembre, 2 004. Huancayo-Perú.

\begin{tabular}{|c|c|c|c|c|c|c|c|c|c|}
\hline \multirow[t]{2}{*}{$\begin{array}{l}\text { Fechas de } \\
\text { evaluaciones }\end{array}$} & \multirow{2}{*}{$\begin{array}{l}\text { Puparios de } L \text {. } \\
\text { huidobrensis } \\
\text { recuperados } \\
\text { NP }\end{array}$} & \multicolumn{2}{|c|}{$\begin{array}{c}\text { Puparios no } \\
\text { viables }\end{array}$} & \multicolumn{2}{|c|}{$\begin{array}{c}\text { Puparios de } L \text {. } \\
\text { huidobrensis } \\
\text { viables }\end{array}$} & \multicolumn{2}{|c|}{$\begin{array}{c}\text { Adultos de } L \text {. } \\
\text { huidobrenis } \\
\text { emergidos }\end{array}$} & \multicolumn{2}{|c|}{$\begin{array}{c}\text { Endo- } \\
\text { parasitoides }\end{array}$} \\
\hline & & $\mathrm{N}^{\circ}$ & $\%$ & $\mathrm{~N}^{\circ}$ & $\%$ & $\mathrm{~N}^{\circ}$ & $\%$ & $\mathrm{~N}^{\circ}$ & $\%$ \\
\hline 20-Ago-04 & 0 & 0 & 0 & 0 & 0 & 0 & 0 & 0 & 0 \\
\hline 27-Ago-04 & 5 & 0 & 0 & 5 & 100 & 3 & 60 & 2 & 40.0 \\
\hline 03-Sep-04 & 3 & 0 & 0 & 3 & 100 & 2 & 67 & 1 & 33.3 \\
\hline $10-$ Sep-04 & 7 & 0 & 0 & 7 & 100 & 6 & 85.7 & 1 & 14.3 \\
\hline 17-Sep-04 & 7 & 0 & 0 & 7 & 100 & 5 & 71 & 2 & 28.6 \\
\hline 24-Sep-04 & 30 & 3 & 10.0 & 27 & 90.0 & 7 & 23 & 20 & 66.7 \\
\hline 01-Oct-04 & 28 & 2 & 7.1 & 26 & 92.9 & 13 & 46.4 & 13 & 46.4 \\
\hline 08-Oct-04 & 112 & 10 & 8.9 & 102 & 91.1 & 47 & 42.0 & 55 & 49.1 \\
\hline $15-O c t-04$ & 117 & 11 & 9.4 & 106 & 90.6 & 67 & 57.3 & 39 & 33.3 \\
\hline 22-Oct-04 & 800 & 107 & 13.4 & 693 & 86.6 & 495 & 61.9 & 198 & 24.8 \\
\hline 29-Oct-04 & 600 & 87 & 14.5 & 513 & 85.5 & 230 & 38.3 & 283 & 47.2 \\
\hline 05-Nov-04 & 681 & 108 & 15.9 & 573 & 84.1 & 215 & 31.6 & 358 & 52.6 \\
\hline 12-Nov-04 & 400 & 86 & 21.5 & 314 & 78.5 & 168 & 42.0 & 146 & 36.5 \\
\hline 19-Nov-04 & 345 & 34 & 9.9 & 311 & 90.1 & 97 & 28.1 & 214 & 62.0 \\
\hline 26-Nov-04 & 342 & 22 & 6.4 & 320 & 93.6 & 41 & 12.0 & 279 & 81.6 \\
\hline 03-Dic-04 & 123 & 23 & 18.7 & 100 & 81.3 & 35 & 28.5 & 65 & 52.8 \\
\hline 10-Dic-04 & 134 & 12 & 9.0 & 122 & 91.0 & 36 & 26.9 & 86 & 64.2 \\
\hline 17-Dic-04 & 123 & 11 & 8.9 & 112 & 91.1 & 37 & 30.1 & 75 & 61.0 \\
\hline
\end{tabular}

\section{Fluctuación poblacional de Halticoptera arduine (Walker)}

Los resultados sobre la fluctuación estacional específica de cada especie de endoparasitoides emergidos de pupas de L. huidobrensis durante las 18 evaluaciones en la estación del mantaro se presentan en el cuadro 3 .

En el cuadro se presentan la fluctuación estacional de Halticoptera arduine $(\mathrm{H})$. En la primera evaluación no se 
registró a esta especie, el 27 de agosto se recuperaron dos individuos que representó el $40 \%$ de parasitismo, en la tercera y cuarta evaluación hubo presencia de la especie. A partir del 24 de setiembre, en la floración del cultivo, se incrementó la temperatura media ambiental y la población de la especie se incrementó, registrándose el porcentaje más alto, el 24 de setiembre con un $50 \%$ que representaba a los 15 individuos recuperados; en los meses de octubre y noviembre durante las evaluaciones se observó un parasitismo que osciló entre $20,5 \%$ al 42,9\% obteniéndose de 12 a 258 individuos, esta repuesta está asociada al desarrollo fenológico de la planta y la mayor población de la mosca minadora. En diciembre (fase de maduración del cultivo) existe un decrecimiento relativo en el porcentaje de parasitismo de 20,3\% a 13,8\% con 17 individuos (03 de diciembre), debido a la menor población de larvas de la mosca minadora.

Estos resultados difieren marcadamente con los obtenidos por Redolfi et al., (1985) quien evaluó en el cultivo de papa en La Molina y registró $22.5 \%$ 12.5\%, $6 \%$ y $6 \%$ el 20 y 30 de setiembre y 10 y 30 de octubre respectivamente. Igualmente difieren con los citados por Sánchez y Redolfi (1988) quienes recuperaron mayor cantidad de adultos de esta parasitoide, así el 27 de setiembre y 11 de octubre registraron 300 y 500 adultos respectivamente en el valle de Cañete en el cultivo de papa.

\section{Fluctuación poblacional de Chrysocharis sp.}

Los resultados sobre la fluctuación estacional específica de cada especie de endoparasitoides emergidos de pupas de $L$. huidobrensis durante las 18 evaluaciones en la estación del Mantaro se presentan en el cuadro 3.

En el cuadro se presentan la fluctuación estacional de Chrysocharis $s p$., la segunda especie de importancia, que se presenta a partir del 24 de setiembre con 5 individuos que representan el 16,7\% de parasitismo, para el 01 de octubre declina la población solo con 01 individuos que representan el 3,6\%. A partir del 08 de octubre el la fase de floración del cultivo, se incrementa el parasitismo en $22,3 \%$ con 25 individuos y esta tendencia es variable, obteniéndose un mayor parasitismo el 26 de noviembre con 35,4\% con 121 individuos en la fase de maduración del cultivo, esta cifra declina en la cosecha .

\section{Fluctuación poblacional de Chrysocharis phytomy- zae (Bretes).}

En el cuadro 3, se muestran los registros de la fluctuación de esta especie. Se observa su presencia a partir del 22 de octubre con 30 individuos que representan un 3,8 \%, en las siguientes evaluaciones se registran porcentajes variables observándose una mayor porcentaje (13\%) con 45 individuos el 19 de noviembre en la fase de maduración, luego declina esta población y en la cosecha sólo se recuperó 13 individuos que representa el 10,6\%.

\section{Fluctuación poblacional de Phaedrotoma sp.}

En el cuadro 3, observamos los registros de la fluctuación de esta especie. Se detectó a partir del 22 de octubre con 10 individuos que representan un 1.3\%. En las siguientes evaluaciones se registran porcentajes variables, así de 13,4\% con 18 individuos el 10 de diciembre en la fase de maduración. A igual que $C h$. phytomyzae la presencia de esta especie se observa en los meses de octubre, noviembre y diciembre en la fase de fructificación y maduración del cultivo. Estos registros difieren marcadamente con los de Espinoza (1999), quien obtuvo un $43.26 \%$ de parasitismo.

\section{Fluctuación poblacional de Diglyphus begini (Ashmead)}

En el cuadro 4, se observa que Diglyphus begini fue el ectoparasitoide de mayor población, registrándose 2 individuos en la fase de macollamiento con una tendencia de incremento en los siguientes días, observándose para el 15 de octubre 56 individuos en la fase de floración e inicio de fructificación, luego la población declina registrándose para la cosecha, 17 de diciembre sólo 8 individuos. Las poblaciones de esta especie ocurrieron durante los meses del desarrollo vegetativo del cultivo con diferentes densidades poblacionales. Respecto a esta especie Mujica (1999) indica que se presenta un alto porcentaje de parasitismo entre 30,7 y $27,9 \%$.

\section{Fluctuación poblacional de Diglyphus websteri (Crawford)}


Esta especie asi como $D$. begini, se presentan en toda las fases fenológicas del cultivo, observándose mayores densidades entre los meses de setiembre y octubre. Estas observaciones no coinciden con lo registrado por Mujica (1 999) quien señala que en octubre se presentaron los más altos porcentajes de parasitismo con promedios de $53.4,41.8,52.9,46.9$ y $54.1 \%$ en cinco diferentes variedades de frijol y los más bajos registros lo obtuvo en noviembre con promedios entre 10.3 a 5\%, Asimismo Redolfi et al., (1988) para el cultivo de frijol en siembras de primavera, registraron para $D$. websteri $57 \%$ de parasitismo. Por otro lado, en el cultivo de papa Redolfi et al., (1985) encontraron que a fines de octubre aumentan las poblaciones de $D$. websteri sobrepasando el $50 \%$ de parasitismo.

Cuadro 4 Ectoparasitoides de I. huidobrensis blanchard de foliolos de haba var. roja UNCP., en la estación experimental del mantaro. Jauja. Periodo: agosto-diciembre, 2 004. Huancayo-Perú.

\begin{tabular}{|c|c|c|c|c|}
\hline \multirow{2}{*}{$\begin{array}{c}\text { Fechas de } \\
\text { evaluaciones }\end{array}$} & \multicolumn{3}{|c|}{ ECTOPARASITOIDES } & \multirow{2}{*}{$\begin{array}{l}\text { Total de } \\
\text { ectopara- } \\
\text { sitoides }\end{array}$} \\
\hline & $\begin{array}{c}\text { Diglyphus } \\
\text { websteri }\end{array}$ & $\begin{array}{l}\text { Diglyphus } \\
\text { begini }\end{array}$ & $\begin{array}{c}\text { Closterocerus } \\
\text { cinctipennis }\end{array}$ & \\
\hline 20-ago-04 & 0 & 0 & 0 & 0 \\
\hline 27-ago-04 & 1 & 2 & 0 & 3 \\
\hline 03-sep-04 & 1 & 2 & 0 & 3 \\
\hline 10 -sep-04 & 4 & 5 & 0 & 9 \\
\hline 17-sep-04 & 8 & 13 & 1 & 22 \\
\hline 24-sep-04 & 25 & 46 & 3 & 74 \\
\hline 01-oct-04 & 12 & 12 & 3 & 27 \\
\hline 08-oct-04 & 10 & 23 & 6 & 39 \\
\hline $15-0 c t-04$ & 28 & 56 & 7 & 91 \\
\hline $22-0 c t-04$ & 2 & 23 & 2 & 27 \\
\hline 29-0ct-04 & 3 & 4 & 4 & 11 \\
\hline 05-nov-04 & 0 & 0 & 0 & 0 \\
\hline 12-nov-04 & 0 & 0 & 0 & 0 \\
\hline 19-nov-04 & 1 & 2 & 2 & 5 \\
\hline 26-nov-04 & 3 & 4 & 0 & 7 \\
\hline 03-dic-04 & 4 & 7 & 1 & 12 \\
\hline 10-dic-04 & 1 & 8 & 0 & 9 \\
\hline
\end{tabular}

\section{Fluctuación poblacional de Closterocerus cinctipen-} nis Ashmead

En el cuadro 4 el registró promedio fue de 1 individuos y el mayor registro ocurrió el 15 de octubre con 7 individuos luego declinó la población sin registrarse en la cosecha. Se observa que las mayores poblaciones de esta especie se presentan en el mes de octubre entre la fase de floración. Estas observaciones fueron similares a lo que registró Mujica (1999) en frijol en siembra de primavera, donde cita de 0.8 a $2.7 \%$ de parasitismo para el mes de noviembre, mientras Redolfi et al., (1986) lo señalan como el segundo ectoparasitoide más importante de $L$. huidobrensis entre setiembre y noviembre.

\section{Comportamiento diapausal de Halticoptera arduine (Walker)}

De las recuperaciones de puparios de la mosca minadora del 10 de diciembre se registró 28 individuos de Halticoptera arduine de los cuales 14 individuos diapausaron 4 meses que representan un 50\%; mientras de la recuperaciones del 17 de diciembre se registró 25 individuos de los cuales 12 individuos diapausaron durante 4 meses y representaron el $48 \%$, sin embargo el menor periodo de diapausa fue de 4,5 meses y se registraron en las recuperaciones del 17 diciembre.

\section{CONCLUSIONES}

- Las mayores poblaciones de L. huidobrensis ocurren en primavera durante los meses de octubre, noviembre y diciembre.

- La mayor riqueza de especies se recupera a partir del 24 de octubre hasta el final de la campaña con siete especies, compuesta de 4 endoparasitoides (Halticoptera arduine, Chrysocharis sp. Chrysocharis phytomyzae y Phaedrotoma sp. y 3 ectoparasitoides (Diglyphus begini, $D$. websteri y Closterocerus cinctipennis).

- Los endoparasitoides más abundantes sobre $L$. huidobrensis son: Halticoptera arduine (Walker) y Chrysocharis sp. mientras los menos frecuentes son Chrysocharis phytomyzae y Phaedrotoma sp. y el ectoparasitoide más abundante es Diglyphus begini y los menos frecuentes son el $D$. websteri y Closterocerus cinctipennis.

- El máximo porcentaje de parasitismo de $H$. Arduine se alcanza al inicio de floración con un $50 \%$.

- H. Arduine presenta estado de diapausa con una duración de 4 a 4,5 meses. 


\section{LITERATURA CITADA}

Chuquillanqui B., W. 1 998. Fluctuación poblacional de insectos en haba cv Pacae Boliviano en la localidad de Ancalá. Tesis para optar el título de Ingeniero Agrónomo. Universidad Nacional del Centro del Perú. Huancayo-Perú. 67 p.

Espinoza S., J. Y Velásquez O., R. 1998. Ciclo biológico de Liriomyza sp. (Diptera; Agromyzidae) plaga del cultivo de haba. Resúmenes. Conv. Nac. Ent. Lima-Perú. 67 p.

Galantini V., L. \& Redolfi de Huiza I. (1 992). Niveles de infestación y parasitismo de Liriomyza huidobrensis en papa cultivada sin aplicación de insecticidas. Rev. per. Ent. 35: 101-106.

Molina S., P. J. 1 990. Evaluación de Liriomyza huidobrensis Blanchard (Diptera: Agromyzidae) y de sus parasitoides en el Cultivo de papa. Tesis para optar el título de Ingeniero Agrónomo. Universidad Nacional Agraria La Molina. Lima-Perú. $120 \mathrm{p}$.

Mujica M., N. C. 1 999. La mosca minadora Liriomyza huidobrensis Blanchard y sus hymenópteros parasitoides en el cultivo de frijol (Phaseolus vulga- ris L. ) bajo condiciones de primavera e invierno en La Molina. Tesis para optar el título de Ingeniero Agrónomo. Universidad Nacional Agraria La Molina. Lima-Perú. 168 p.

Sánchez V. G. y Redolfil De Huiza I. 1988. Liriomyza huidobrensis y sus parasitoides en papa cultivada en Rimac y Cañete 1986. Rev. Ent. 31:110112.

Tejada H., G. H. 1 998. Fluctuación poblacional de Liriomyza huidobrensis Blanchard (Diptera : Agromyzidae), sus parasitoides y de Prodiplosis longifila (Gagné) (Diptera: Cecidomyiidae), en campos de alfalfa tratados con insecticidas en la zona baja del valle de Lurín. Tesis para optar el grado de Magister Scientiae. Universidad Nacional Agraria La Molina. Lima-Perú. 163 p.

Valverde C., A. y Paucarchuco T., T. 2 000. Dinámica poblacional de la mosca minadora Liriomyza huidobrensis sobre habas en el Valle del Mantaro. Informe final de investigación. Universidad Nacional del Centro del Perú .19 p.

Wille T., J. E. 1 952. Entomología agrícola del Perú. Segunda edición. Imp. Aramburú. Raygada Hnos. S.A. Junta de Sanidad Vegetal-Ministerio de Agricultura Lima-Perú. 543 p. 